\title{
Applicability of a 4 inputs ANN model for ETo prediction in coastal and inland locations
}

\author{
P. Martí, A. Royuela, J. Manzano \& G. Palau \\ Valencian Centre for Irrigation Studies CVER, \\ Polytechnic University of Valencia, Spain
}

\begin{abstract}
Artificial Neural Networks (ANNs) are simplified models of the central nervous system that can be used as effective tools to model nonlinear problems. This paper reports the study of the applicability of an ANN-based ETo predicting-model in different geographical contexts of the Valencia region. The proposed model only demands the measurement of the maximum and minimum daily air temperatures and the calculation of extraterrestrial radiation and daylight hours. The model provides acceptable approximations of Penman-Monteith ETo values, better than already existing ETo predicting tools (Hargreaves), for coastal locations, where the sea contributes to hinder drastic climatological fluctuations. Nevertheless, the mapping capability of this model in other places with higher indexes of continentality is to be questioned. Furthermore, the possibility of achieving good ETo predictions in different inland locations to those used to train the network might be looked with uncertainty, because of the local uniqueness of the complex relationships between temperature and ETo. On the other hand, models trained in coastal locations might be preferable to carry out predictions in inland locations. The proposed 4-inputs ANN can be useful and preferable to other methods when ETo models which demand a high number of variables cannot be used.
\end{abstract}

Keywords: ETo prediction, artificial neural networks, maximum and minimum temperatures, index of continentality.

\section{Introduction}

The precise quantification of cropping evapotranspiration has become a very important task, due to the current water shortage and the subsequent rise of water price. On the other hand, the already existing models that provide precise enough 
ETo-predictions demand a high number of climatic inputs for their performance. For both reasons, the development of more efficient ETo predicting tools for those cases where only scan climatic data are available turns into a task of great relevance. Between the most common applications of ANNs we distinguish: constraint satisfaction, control, data compression, diagnostics, forecasting, general mapping, multisensory data fusion, optimization, pattern recognition and risk assessment (Patterson [1]). Several studies have been carried out taking advantage of the input-output mapping capability of neural networks for ETo modelling (Sudheer et al [2], Kumar et al [3], Trajkovic et al [4], Zanetti et al [5]).

ANNs are massively parallel distributed processors made up of simple processing units, which have a natural propensity for storing experimental knowledge and making it available for use (Haykin [6]). An ANN is configured for a specific application, such as pattern recognition or data classification, through a learning process. Learning in biological systems involves adjustments to the synaptic connections that exist between the neurons. Because of their nonlinear structure, ANNs are able to capture more complex properties of the studied data than the traditional statistical techniques (Galvão et al [7]). Moreover, the main advantage of neural networks in comparison to conventional methods is that they do not require detailed information on the physical processes of the system (Sudheer et al [2]).

In the last year, Zanetti et al [5] used artificial neural networks for estimating reference evapotranspiration (ETo) as a function of the maximum and minimum air temperatures, extraterrestrial radiation and daylight hours in a coastal county of Rio de Janeiro state, Campos dos Goytacazes. While the first two variables were to be measured, the other two ones were calculated as a function of the local latitude and Julian data. Thus, the proposed model only depends on the measurement of two climatic variables, what can be carried out with a simple thermometer. On the other hand, the authors trained their model with Campos dos Goytacazes climatic series and tested the resulting ANN with Campos dos Goytacazes and a near location, Vicoça, data sets, achieving performance indexes of 0.875 and 0.838 , respectively, which correspond to 'excellent' $(c>0.85)$ and 'very good' $(0.76<\mathrm{c}<0.85)$ performances, according to the classification proposed by Camargo et al [8].

The high interest of the idea proposed in that paper and the local presence of an extensive weather stations network, with availability of a wide range of climatic variables, encouraged the authors to carry out an analogous study in the autonomous Valencia region, in the Mediterranean coast of Spain. Moreover, the authors have focussed their study on the applicability of this ANN-model in different geographical contexts, analysing the possible effect of the sea on the performance errors of the ANNs.

\section{Materials and methods}

\subsection{Climatic data}

The historical series of the climatic variables used in this study were obtained from the weather stations of the Valencian Institute for Agricultural 
Investigations (IVIA) in the municipalities of Sagunt (latitude $39^{\circ} 38^{\prime} 57^{\prime \prime} \mathrm{N}$, longitude $0^{\circ} 17$ ' 33' $\mathrm{W}$, altitude $33 \mathrm{~m}$ ), Vila Joiosa (latitude $38^{\circ} 31^{\prime} 46^{\prime}$ ' $\mathrm{N}$, longitude $0^{\circ} 15^{\prime} 19^{\prime}$ ' $\mathrm{W}$, altitude $138 \mathrm{~m}$ ), Camp de Mirra (latitude $38^{\circ} 40^{\prime} 49^{\prime}$ ' $\mathrm{N}$, longitude $0^{\circ} 46^{\prime} 18^{\prime}, \mathrm{W}$, altitude $627 \mathrm{~m}$ ) and Novelda (latitude $38^{\circ} 22^{\prime} 42^{\prime}$ ' $\mathrm{N}$, longitude $0^{\circ} 44^{\prime} 49^{\prime}, \mathrm{W}$, altitude 244m) - figure 1 - from January 2001 to December 2006 for Sagunt and Vila Joiosa and from January 2000 to December 2005 for the others. The daily values of maximum, minimum and average temperature, average and maximum wind speed, relative air humidity, solar radiation, sunshine duration were collected by an automatic meteorological station. In Sagunt and Vila Joiosa ANNs the daily values from 2005 were used for network testing and the data series of the other five years were used for training. In Novelda and Camp de Mirra ANNs the daily values from 2004 were used for testing and the data series of the other five years were used for training. The choice of 2004 as test year was due to the breakdowns that took place during several days of 2005 in Novelda and Camp de Mirra stations. Nevertheless it seems that this fact can't interfere in the sense of the comparison results carried out, since all the data series involved in the study can be considered as normal from a climatic point of view, without sharp or noticeable changes during these years.
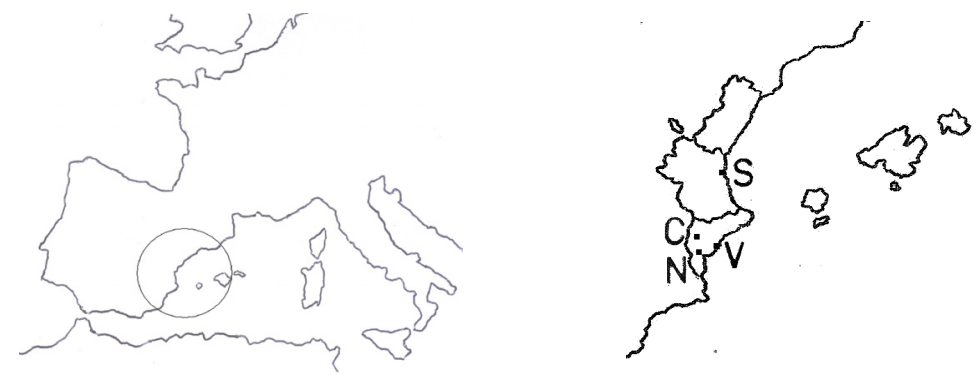

Figure 1: Map of the meteorological station locations: Sagunt (S), Vila Joiosa (V), Novelda (N) and Camp de Mirra (C).

\subsection{Methods}

A network is divided into layers. The input layer consists of just the inputs to the network. Then follow the hidden layers, which are middle units placed in parallel and consist of any number of neurons. Each neuron performs a weighted summation of the corresponding inputs, which then passes an activation function, also called neuron function. After the learning process the knowledge represented in the model remains stored in the weights. All used ANN neurons were configured, based on the model by Haykin [6]. The first subscript of the synaptic weight $w_{k j}$ refers to the neuron in question and the second one refers to the input sign of the synapse to which the weight refers.

For each neuron, the activation or transfer function is applied to the corresponding summing junction, sum of all input signals weighted by the 
corresponding synaptic weights (same first subscript), in order to limit its amplitude to values between two asymptotes and to add nonlinearity to the model. On the other hand, the bias has the effect of increasing or reducing the net entrance value of the activation function. The sigmoidal function is the most common form of transfer function, it is a continuous function that varies between two asymptotic values, usually 0 and 1 or -1 and 1 . Thus, the hyperbolic tangent sigmoid and the lineal functions were adopted for the intermediate and the output layers, respectively.

The used architectures correspond to multilayer feed-forward networks with back-propagation. Thus, they are feed-forward (the signal spreads layer by layer forward), fully-connected hierarchical networks that use differentiable activation functions and supervised training which involves an iterative procedure for minimization of the error function (performance function).

During the learning phase, input patterns are presented to the network in some sequence. Each training pattern is propagated forward until an output pattern is computed. The computed output is then compared to a desired or target output and an error value is determined. The errors are used as inputs to feedback connections from which adjustments are made to the synaptic weights layer by layer in a backward direction. The hidden layer weights are adjusted using the errors from the subsequent layer. The process is repeated a number of times for each pattern in the training set until the fixed criterion is fulfilled or until some limit is reached in the number of training iterations completed. The early stopping procedure was used as the criterion to finalize the training. That way, training data series were divided into two groups: one for learning/parameter estimation and one for cross-validation. According to this method, when the chosen error, of the cross validation set was lower than its value in the previous iteration, the training of the network proceeded; if not, the training was finished. Training was carried out under supervision with the Levenberg-Marquardt algorithm. Neural network minimization problems are often very ill-conditioned, that is, the Hessian is often ill-conditioned. This makes the minimization problem harder to solve, and for such problems, the Levenberg-Marquardt algorithm is a good choice.

Instead of following the methodology applied by Zanetti et al [5], where several architectures with a fixed number of neurons per layer were defined and tested, the authors have developed a general procedure which allows each time to choose the optimum architecture from a set that considers up to three hidden layers with one up to $\mathrm{n}$ neurons each, where the different hidden layers present always the same number of neurons. Moreover, each architecture is calculated $r$ times and the corresponding average ANN parameters are considered. For this purpose, Matlab 2007a was used. When dealing with the creation of predicting tools based on ANNs, the corresponding statistical performance parameters should stem from an average of several training-test processes in order to avoid the effects derived from the random assignment of the weights when the training algorithm is initialized. In our case, maximum number of neurons per layer and number of repetitions were fixed in 20 each. The developed program selects the architecture that provides the best average performance and simulates the test 
data series of every weather station, in order to check the validity of the ANN model outside the location that has been used to train the model.

The selected training parameters are summed up in the following table.

Table 1: $\quad$ Parameters used in the training process.

\begin{tabular}{|c|c|}
\hline peformance function & MSE \\
\hline maximum number of epochs to train & 100 \\
\hline performance goal & 0 \\
\hline maximum validation failures & 5 \\
\hline minimum performance gradient & $1 \mathrm{E}-10$ \\
\hline initial mu & 0.001 \\
\hline mu decrease factor & 0.1 \\
\hline mu increase factor & 10 \\
\hline maximum mu & $1 \mathrm{E}+10$ \\
\hline maximum time to train & inf \\
\hline
\end{tabular}

On the other hand, the ANN estimations were compared with Hargreaves ETo predictions and several associated statistical performance indicators were computed. Thus,

$$
M S E=\frac{\sum_{i=1}^{n}\left(y_{m_{i}}-y_{e_{i}}\right)^{2}}{n}
$$

Where MSE is the mean squared error, $y_{e}$ is the desired or target output and $y_{m}$ the computed output.

$$
d=1-\frac{\sum_{i=1}^{n}\left(y_{m_{i}}-y_{e_{i}}\right)^{2}}{\sum_{i=1}^{n}\left(\left|y_{m_{i}}-\bar{y}_{m}\right|+\left|y_{e_{i}}-\bar{y}_{e}\right|\right)^{2}}
$$

where $\mathrm{d}$ is the adjustment coefficient, $\bar{y}$ is the average of the corresponding $\mathrm{y}$ values, $m$ and e refer to model and experimental values, respectively.

$$
c=d \cdot r
$$

where $\mathrm{c}$ is the performance index and $\mathrm{r}$ is the correlation coefficient.

\section{Results and discussion}

The accuracy of each ANN prediction was measured by means of $\mathrm{c}$ and MSE, calculated for the ANN/Hargreaves predictions and the corresponding Penman Montheith values. The statistical outputs of the created matlab program are gathered in tables 2 and 3. Vila Joiosa and Sagunt present better performance indicators than Novelda and Camp de Mirra. While Novelda shows unacceptable associated indicators, the two coastal weather stations achieve even better 
performances than the ones presented by Zanetti et al [5] in Campos dos Goytacazes. As can be seen in table 2, the most suitable architecture varies with the studied location, although the analysis of the results that provide these average parameters reveals that one single hidden layer might be enough to represent the nonlinear relationship between the climatic inputs and the ETo, as was concluded by Kumar et al [3] and Zanetti et al [5]. In addition, the ANN model seems to generate more accurate predictions than the Hargreaves one. On the other hand, table 3 sums up the parameters that quantify the potential of these ANNs to forecast ETo outside the place where the model has been trained.

Table 2: $\quad$ Average indicators of optimum architecture and Hargreaves model indicators $\left[\mathrm{MSE}\right.$ in $\left.(\mathrm{mm} / \mathrm{day})^{2}\right]$.

\begin{tabular}{|c|c|c|c|c|c|c|}
\hline \multicolumn{5}{|c|}{ optimum average architectures } & \multicolumn{2}{c|}{ Hargreaves model } \\
\hline $\begin{array}{c}\text { training } \\
\text { location }\end{array}$ & $M S E$ & $c$ & $\begin{array}{c}\text { number of } \\
\text { layers }\end{array}$ & $\begin{array}{c}\text { neurons per } \\
\text { layer }\end{array}$ & MSE & $c$ \\
\hline C. Mirra & 0.7232 & 0,8401 & 1 & 8 & 1.0446 & 0.8631 \\
\hline Sagunt & 0.3299 & 0,9105 & 2 & 19 & 0.4693 & 0.8761 \\
\hline Novelda & 2.2913 & 0,5314 & 1 & 17 & 1.6894 & 0.5437 \\
\hline Vila Joiosa & 0.2290 & 0,9289 & 2 & 7 & 0.2686 & 0.9254 \\
\hline
\end{tabular}

Table 3: MSE and performance index of the tested data sets [MSE in $\left.(\mathrm{mm} / \text { day })^{2}\right]$.

\begin{tabular}{|c|c|c|c|c|c|c|c|c|}
\hline & \multicolumn{7}{|c|}{ test location } \\
\cline { 2 - 10 } & \multicolumn{2}{|c|}{ C. Mirra } & \multicolumn{2}{|c|}{ Sagunt } & \multicolumn{2}{c|}{ Novelda } & \multicolumn{2}{c|}{ Vila Joiosa } \\
\hline $\begin{array}{c}\text { training } \\
\text { location }\end{array}$ & $M S E$ & $c$ & $M S E$ & $c$ & $M S E$ & $c$ & $M S E$ & $c$ \\
\hline C. Mirra & 1,418 & 0,843 & 0,438 & 0,895 & 2,496 & 0,520 & 0,373 & 0,914 \\
\hline Sagunt & 1,990 & 0,72 & 0,787 & 0,806 & 3,331 & 0,389 & 1,403 & 0,719 \\
\hline Novelda & 0,899 & 0,863 & 0,810 & 0,822 & 2,324 & 0,525 & 0,512 & 0,874 \\
\hline V. Joiosa & 0,819 & 0,852 & 0,479 & 0,870 & 1,675 & 0,536 & 0,289 & 0,913 \\
\hline
\end{tabular}

Thus, the results corresponding to Novelda test series show very poor predicting potential of the model for this location. Nevertheless, attending to the performances achieved with the rest of test locations, especially in the similar inland weather station, Camp de Mirra, and the results obtained when the model is trained in Novelda and tested outside (for example in Vila Joiosa $\mathrm{c}=0.87$ and MSE $\left.=0.51(\mathrm{~mm} / \text { day })^{2}\right)$, it might be concluded that Novelda test data set could contain errors or lowly reliable data. Penman-Monteith ETo estimations and the corresponding ANN predictions for the case where the model is trained and tested in the same location are presented in figure 2. It can be seen that Novelda PM-ETo doesn't show a clear annual trend like the other weather stations. In this way, as the measurement of a considerable number of climatic variables is 

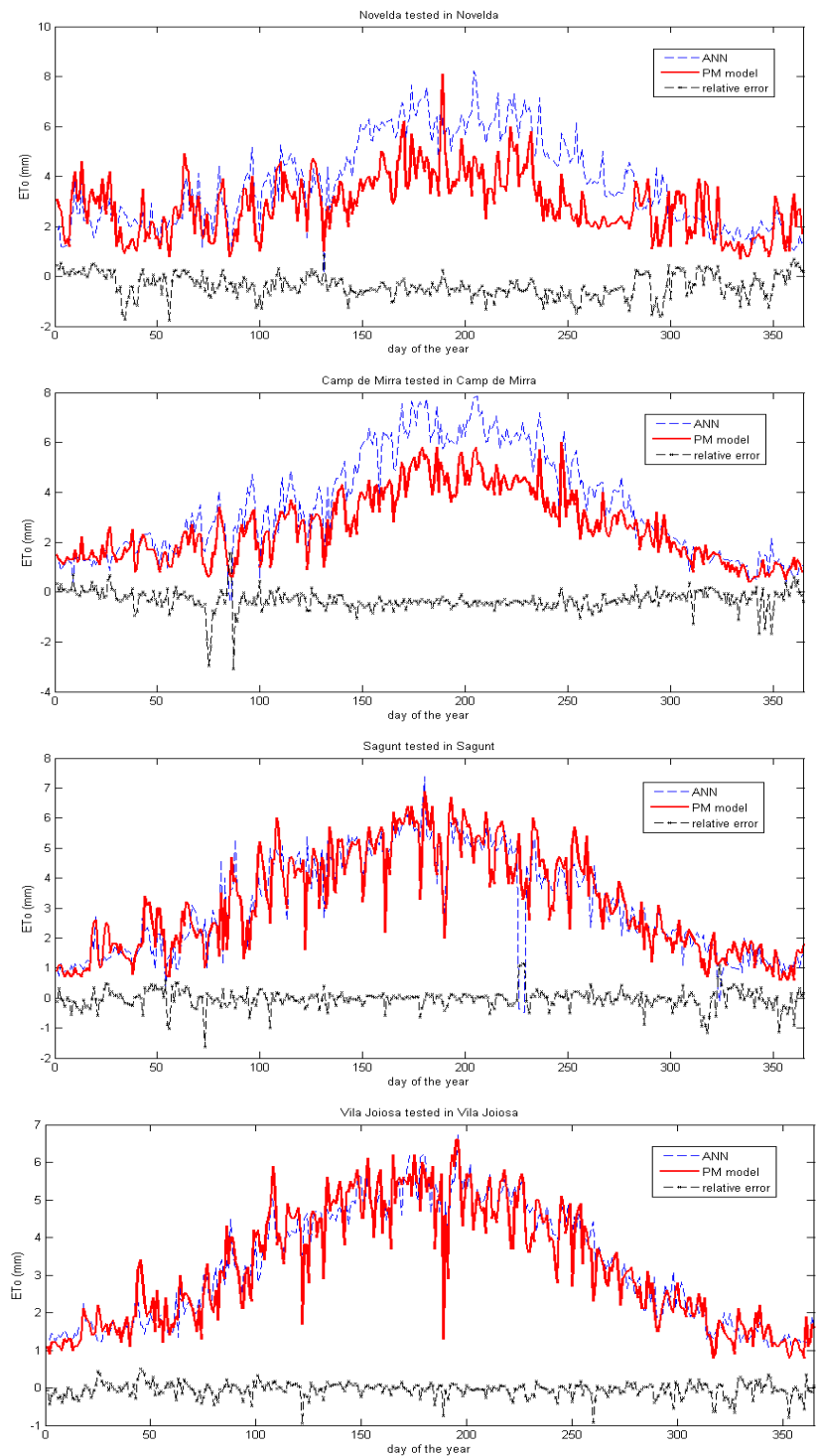

Figure 2: $\quad$ Comparison between the Penman Monteith ETo estimation and the 4 inputs ANN prediction in Novelda, Camp de Mirra, Sagunt and Vila Joiosa.

necessary for the application of the Penman-Monteith model, some errors might have been taking place in the climatic data acquisition during the test year. In contrast, neglecting the parameters that derive from the use of Novelda test set, the performance indexes that are reached exceed 0.8-0.85, with the exception of the model that is trained in Sagunt and tested in Sagunt and Vila Joiosa. Nevertheless, though the fact that this parameter might be acceptable, the mapping potential of 
this model descends outside the training location, as can be seen through the increase of the corresponding MSE. However, it's possible to find cases where the model seems to fit in some way. Thus, when the model is trained in Camp de Mirra and tested in Sagunt and Vila Joiosa the mean square error reaches 0.43 and 0.37, respectively, and when it's trained in Vila Joiosa and tested in Sagunt it comes to 0.47 . Furthermore, it turns out to be peculiar in some sense the fact that for some locations a model that is trained outside provides better performance indicators (for example, Novelda and Vila Joiosa models when they are tested in Camp de Mirra or Vila Joiosa and Camp de Mirra models when they are tested in Sagunt). The peak of Sagunt ANN curve has turned out to be due to the presence of four false values of the minimum temperature during august.

In order to characterize the 4 inputs model validity in relationship with some climatic indicator of the studied location, three factors have been proposed for each location as alternatives to the existing indexes of continentality. These factors pretend to quantify in some way the scale of thermal oscillation that takes place for a year data set. Therefore, $\alpha$ has been defined as the annual average of the daily thermal amplitude divided by the maximum annual thermal oscillation, $\beta$ is the annual amount of the daily thermal amplitude divided by the maximum annual thermal oscillation and $\gamma$ is obtained by multiplying factor $\alpha$ by the minimum distance from the studied location to the sea. The values corresponding to the studied weather stations are gathered in table 4 .

Table 4: $\quad$ Proposed thermal oscillation indexes for the test data sets.

\begin{tabular}{|c|c|c|c|}
\hline \multicolumn{4}{|c|}{ Thermal oscillation indexes } \\
\hline location & $\alpha$ & $\beta$ & $\gamma$ \\
\hline Camp de Mirra & 0,266 & 97 & 11,3 \\
\hline Sagunt & 0,225 & 82 & 1,13 \\
\hline Novelda & 0,283 & 103 & 6,72 \\
\hline Vila Joiosa & 0,249 & 90 & 0,31 \\
\hline
\end{tabular}

Consequently, it might be convenient to relate a higher predicting potential of the 4 inputs model with a lower index of thermal oscillation. Although Novelda ETo test data are suspicious to contain errors, its temperature series might be correctly measured. As it could be foreshadowed, coastal locations present lower thermal oscillation due to the moderating role played by the sea. Parameters $\alpha$ and $\beta$ assign a higher thermal oscillation to Novelda towards Camp de Mirra, although this one is further away from the sea. The weather station where the model shows the best performance is Vila Joiosa, the location with the lowest thermal oscillation indexes.

\section{Conclusions}

In this paper, the applicability of the 4 inputs ANN model proposed by Zanetti et al [5], which considers maximum and minimum daily temperatures, extraterrestrial radiation and daylight hours, has been tested for ETo prediction 
in four weather stations of the Valencia region. Moreover, a general procedure to select the most suitable ANN configuration for any study case has been developed with Matlab.

The results achieved by Zanetti et al [5] for their 4-inputs ANNs are due to the fact that the climatic series data used for training and testing the model came from a coastal location, where the sea plays a decisive role. The mapping capability of this model in other places with more marked climatological fluctuations is to be questioned. Therefore, the authors suggest a restriction in the applicability of this model for climatic zones with low indexes of continentality and propose the creation of geographical maps for those places where this model is valid taking into consideration the corresponding indexes of continentality, which should quantify the scale of thermal amplitude that takes place for an average year data set. For the current study cases Pérez Cueva [9] calculated the following Gorczinsky (G.i.) and Conrad (C.i.) indexes: Novelda and Camp de Mirra (G.i. between 22 and 26, C.i. between 20 and 24) and Sagunt and Vila Joiosa (G.i. and C.i. between 16 and 17). As these ones only take into account the latitude and the average annual thermal amplitude, the authors have seen convenient to propose other parameters which considered the distance to the sea and the relationship between daily and annual thermal oscillations.

Furthermore, the authors look with uncertainty the possibility of achieving good ANN ETo predictions in different places to those used to train the network, because of the local uniqueness of the complex relationships between temperature and ETo. Therefore, further studies should be carried out in order to establish more drastic trends of the model generalization. Taking into account the air relative humidity and/or the daily average wind speed as input variables would considerably rise the potential of the ANN to estimate ETo in other places. The current 4-inputs model can be useful and preferable to other methods (e.g. Hargreaves) when ETo models which demand high number of variables can't be used, for instance, when a breakdown of the weather station takes places.

Finally, the authors suggest the interest of defining general procedures to obtain optimized architectures for each case. To achieve this objective the application of genetic algorithms can result very useful, as it was seen by Ritchie et al [11].

\section{References}

[1] Patterson, D.W., (ed). Artificial Neural Networks. Theory and Applications, Prentice Hall: Singapore, 1996.

[2] Sudheer K.P., Gosain A.K. \& Ramasastri K.S., Estimating actual evapotranspiration from limited climatic data using neural computing technique. Journal of Irrigation and Drainage Engineering, 129 (3), pp. 214-218, 2003.

[3] Kumar M., Raghuwanshi, N.S., Singh R., Wallender W.W. \& Pruitt W.O., Estimating evapotranspiration using artificial neural network. Journal of Irrigation and Drainage Engineering, 128 (4), pp. 224-233, 2002. 
[4] Trajkovic S., Todorovic B. \& Stankovic M., Forecasting of reference evapotranspiration by artificial neural networks. Journal of Irrigation and Drainage Engineering, 129 (6), pp. 454-457, 2003.

[5] Zanetti S.S., Sousa E.F., Oliveira V.P.S., Almeida F.T. \& Bernardo S., Estimating evapotranspiration using artificial neural network and minimum climatological data. Journal of Irrigation and Drainage Engineering, 133 (2), pp. 83-89, 2007.

[6] Haykin, S., (ed). Neural Networks. A comprehensive foundation, Prentice Hall International Inc: New Jersey, 1999.

[7] Galvão, C.O., Valença, M.J.S., Vieira, V.P.P.B., Diniz, L.S., Lacerda, E.G.M., Carvalho, A.C.P.L.F. \& Ludemir, T.B. (eds). Sistemas inteligentes: Aplicações a recursos hídricos e ciencias ambientais, Universidade Federal do Rio Grande do Sul: Porto Alegre, 1999.

[8] Camargo, A.P. \& Sentelhas, P.C., Avaliação do desempenho de diferentes métodos de estimativa da evapotranspiração potencial no Estado de São Paulo, Brazil. Revista Brasileira de agrometeorologia, 5(1), pp. 89-97, 1997.

[9] Pérez Cueva A.J., (ed). Atlas Climàtic de la Comunitat Valenciana (19611990), Conselleria d'Obres Públiques, Urbanisme i Transports. Sèrie Publicacions de Divulgació Tècnica. Col.lecció Territori: Valencia, 1994.

[10] Martin Vide J., (eds) Fundamentos de climatología analítica, Editorial Síntesis: Madrid, 1991.

[11] Ritchie, M.D., White, B.C., Parker, J.S., Hahn, L.W. \& Moore, J.H., Optimization of neural network architecture using genetic programming improves detection and modelling of gene-gene interactions in studies of human diseases. BMC Bioinformatics, 4(28), pp. 1-14, 2003. 